\title{
Effect of Aromatic Essential Oil of Lavender on the Electrical Activity of Healthy Girls' Heart during Exercise
}

\section{ARTICLE INFO}

\section{Article Type}

Original Research

\section{Authors}

Torabi M. ${ }^{1} M S c$,

Moharamzadeh S.* MSc

Ebrahim Kh. ${ }^{2} \mathrm{PhD}$

How to cite this article
Torabi M, Moharamzadeh S, Ebr-
ahim Kh. Effect of Aromatic Ess-
ential Oil of Lavender on the El-
ectrical Activity of Healthy Girls'
Heart during Exercise. Horizon
of Medical Sciences. 2017;23(2)
$: 99-104$.

*Sport Physiology Department, Sport Science Faculty, Shahid Rajaii Teacher Teaching University, Tehran, Iran

${ }^{1}$ Sport Physiology Department, Sport Science Faculty, University of Guilan, Rasht, Iran

${ }^{2}$ Sport Physiology Department, Sport Science Faculty, Shahid Beheshti University, Tehran, Iran

\section{Correspondence}

Address: Sport Physiology Department, Sport Science Faculty, Shahid Rajaii Teacher Teaching University, Shabanlou Street, Lavizan, Tehran, Iran. Postal Code: 1678815811

Phone: +98 (21) 26329095

Fax: +98 (21) 26329095

s.moharamzadeh@srttu.edu

\section{Article History}

Received: February 12, 2016

Accepted: November 14, 2016

ePublished: March 25, 2017

\section{A B S T R A C T}

Aims Some of the herbal supplementations consumed by the athletes in order to improve their athletic functions are selected to affect the cardio-vascular system. The aim of the study was to investigate the aromatic effects of lavender essential oils on the heart electrical functions during exercises in the healthy girls.

Materials \& Methods In the repeated pretest-posttest semi-experimental study, 9 active healthy girls studying sport sciences in Shahid Rajaei University were studied in 2015. The subjects were selected via purposeful sampling method. As counter balance, either ethanol soaked cotton or lavender oil essence were exposed to each subject, and the Conconi test was conducted as a sport activity. Both electrocardiogram waves and blood pressure data were recorded before the activities and immediately after and one minute after the end of the activity (recovery). Data were analyzed by SPSS 20 software using repeated ANOVA and student T tests.

Findings Immediately after the test, the systolic blood pressure in experimental group was significantly higher than control group $(\mathrm{p}<0.05)$. In addition, the inhalation of the lavender oil in experimental group increased $\mathrm{R}$ wave height during the final moments of exercises compared to control group $(\mathrm{p}<0.05)$. Nevertheless, the systolic blood pressure, $\mathrm{T}$ wave height, and R-R and Q-T distances did not significantly change in response to the exercises. Conclusion During the sport activities, the inhalation of lavender affects the heart ventricular function, as well as the heart beat and the systolic blood pressure.

Keywords Lavender Oil; Electrocardiogram; Exercise; Girls

\section{I T A T I O N L IN KS}

[1] Adaptation to exercise-induced oxidative stress ... [2] Effects of endurance training on resting and post-exercise cardiac autonomic ... [3] Introduction: eligibility recommendations for competitive ... [4] Recommendations for the standardization and interpretation of the electrocardiogram: part I: The electrocardiogram and its technology a scientific statement from the American Heart Association Electrocardiography and Arrhythmias Committee, Council on Clinical ... [5] Effect of moderate exercise on the electrocardiogram in healthy young ... [6] Biomarkers for effects of fatigue and stress on performance ... [7] Task force 9: Drugs and performance-enhancing ... [8] The effect of garlic supplementation on aerobic performance ... [9] Biological activities of lavender ... [10] The effect of Lavender essence via inhalation method on ... [11] Effect of aromatherapy with lavender ... [12] Comparison of Lavandula officinalis tincture and imipramine in the treatment of mild to moderate depression ... [13] Anti-inflammatory and analgesic properties of the leaf extracts and essential oil of Lavandula ... [14] Cardioprotective effects of essential oil of Lavandula angustifolia on isoproterenol-induced acute myocardial infarction ... [15] Sensing an improvement: an experimental study to evaluate the use of aromatherapy, massage and periods of rest in an ... [16] Lavender aromatherapy in recovery from ... [17] Pleasant odors attenuate the blood pressure increase during rhythmic handgrip in ... [18] Practical ECG for exercise science and sports ... [19] A new system of multiple-lead exercise ... [20] Exercise-induced changes in R wave amplitude and heart rate in normal ... [21] High blood glucose level on hospital admission and poor neurological recovery after cardiac ... [22] Guyton and Hall textbook of medical ... [23] Relaxation effects of lavender aromatherapy improve coronary flow velocity reserve in healthy men evaluated by transthoracic Doppler ... [24] Use of the electrocardiogram in acute myocardial ... [25] Cardiac biochemical, electrical and functional alterations in a group of untrained males after one bout of maximal isometric ... 
اولين بار توسط سيمونسون در سال سمها19 مطرح شد [5]. همجنين

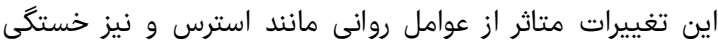

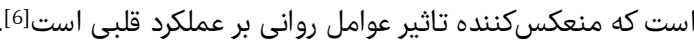

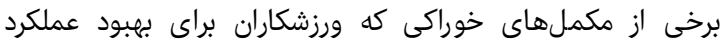

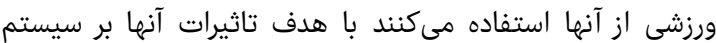

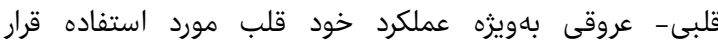

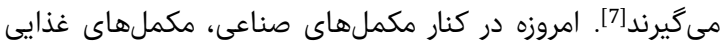

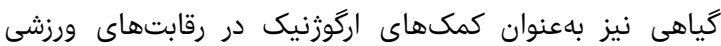
استفاده مى شوند[8]. اسطوخودوس يا لاواندر (Lavander) كياه

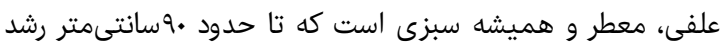

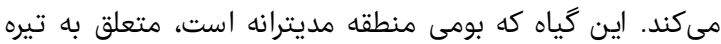

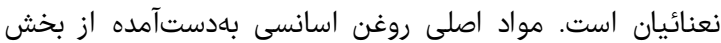

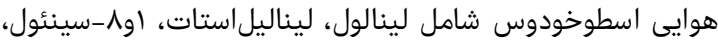

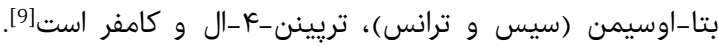

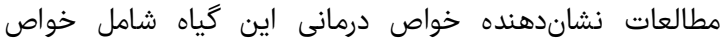

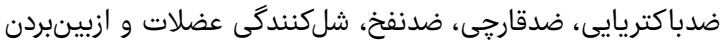

درد هستند[10].

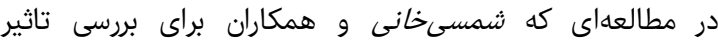

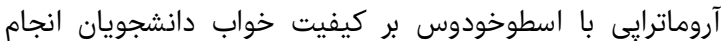

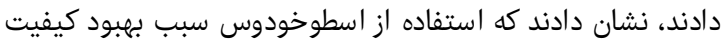

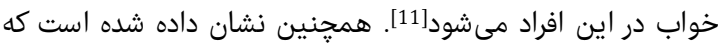

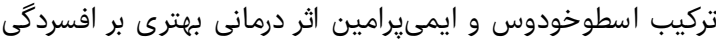

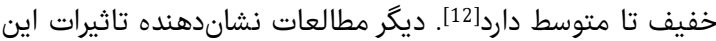

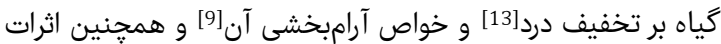
محافظتى آن بر سيستم قلبى - عروقى [14] هستئند.

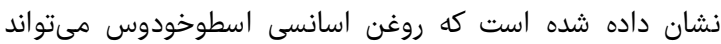

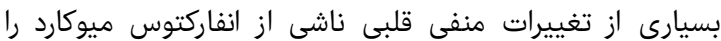

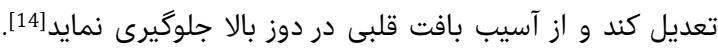

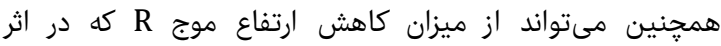

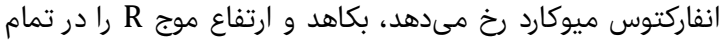

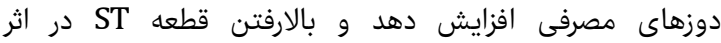

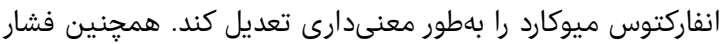

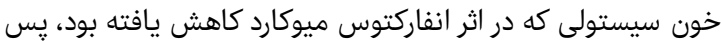

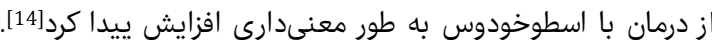

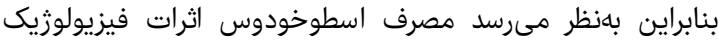

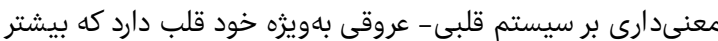

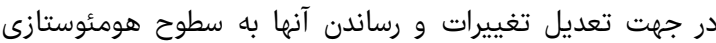

فعاليت ورزشى از جمله شرايط تنشزا براى بدن محسوب مى شيود

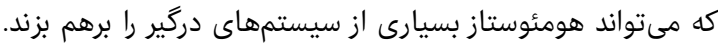

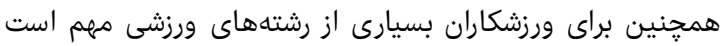

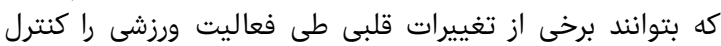

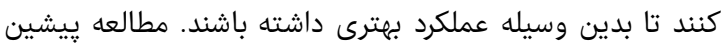

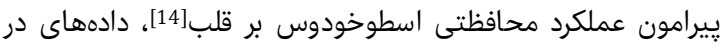

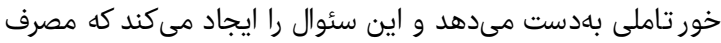

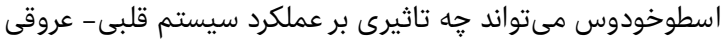

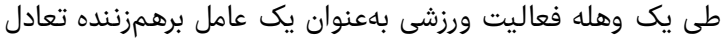

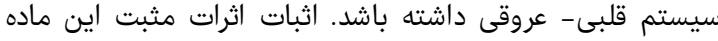

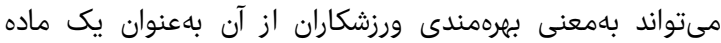

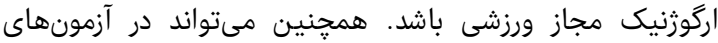

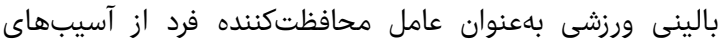
احتمالى مورد استفاده قرار گيرد.

دوره rr.، شماره r، بهار 7qسו

\section{تاثير روغن آروماتيك اسانسى اسطوخودوس بر

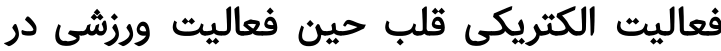 دختران سالم}

\author{
MSc M ميثم ترابى \\ كَروه فيزيولوزى ورزشى، دانشكده علوم ورزشى، دانشكاه كَيلان، رشت، ايران \\ MSc " سودا محرمزادهي \\ كروه فيزيولوزى ورزشى، دانشكده علوم ورزشى، دانشكاه تربيت دبير شهيد

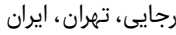 \\ خرو ابراهيم PhD
}

كروه فيزيولوزى ورزشى، دانشكده علوم ورزشى، دانشكاه شهيد بهشتى، تهران، ايران

جكيده

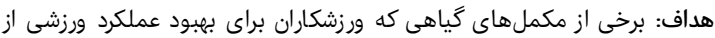

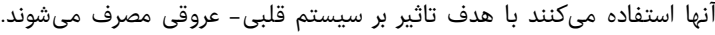

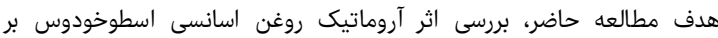

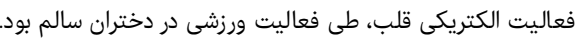

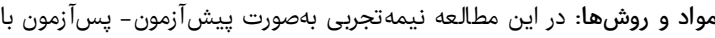

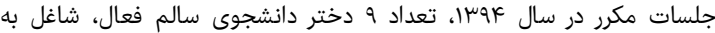

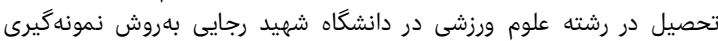

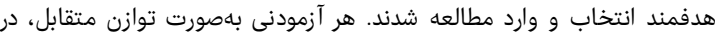

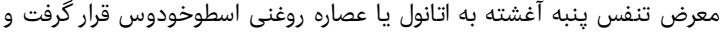

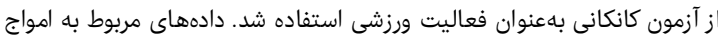

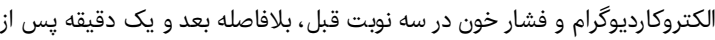

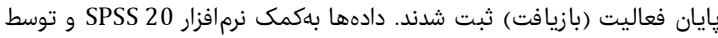

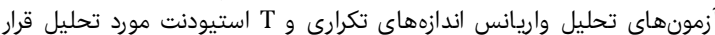

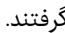

يافتهها: فشار خون سيستولى بلافاصله يّ إز از فعاليت ورزشى، در گروه آزمون

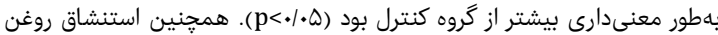

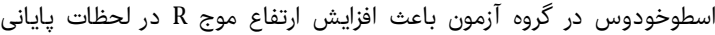

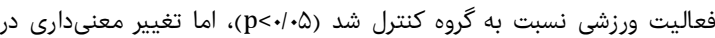

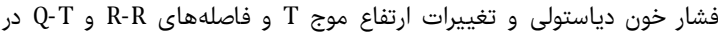

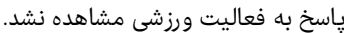

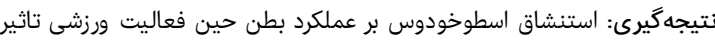

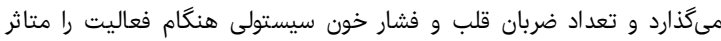

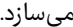
كليدوازمها: روغن اسطوخودوس، الكتروكارديوكرام، فعاليت ورزشى، دختران

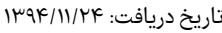

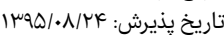
'نويسنده مسئول: s.moharamzadeh@srttu.edu

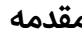

فعاليت بدنى از جمله عوامل استرسزا براى بدن محسوب مى شئهود

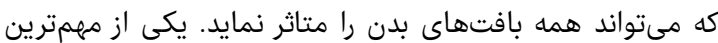

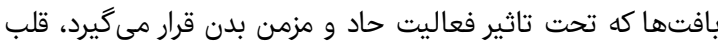

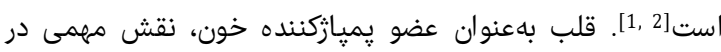

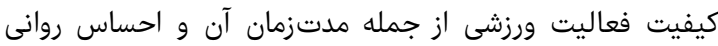

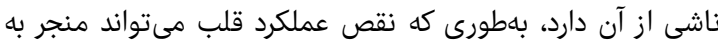

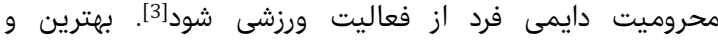

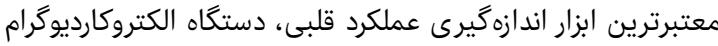

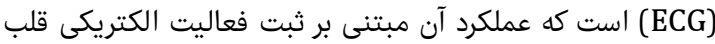

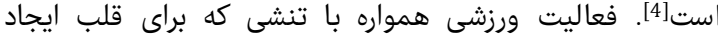

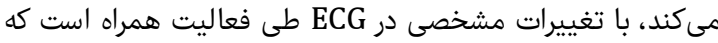
فصل نامه علمى - يزوهشى افق دانش 


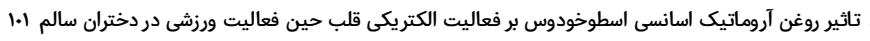

آمارى تحليل واريانس اندازههاى تكرارى و T T استيودنت استفادئ شد و دادهها بهوسيله نرمافزار SPSS 20 آناليز شدند.

يافتهها

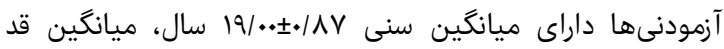

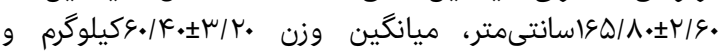
حداكثر اكسيثن مصرفى.ه/

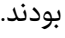

فعاليت ورزشى در هر دو گروه كنترل و آزمون، موجب افزايش

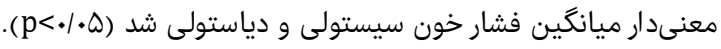

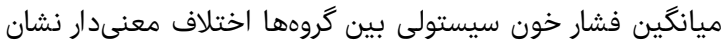

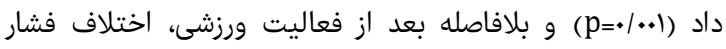

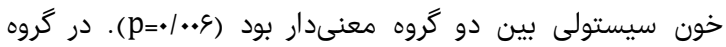

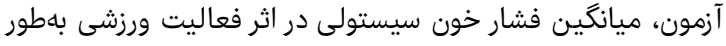

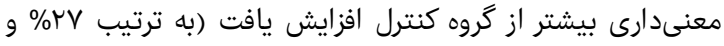

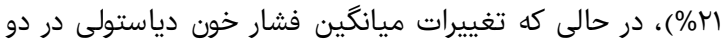

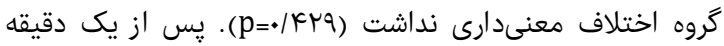

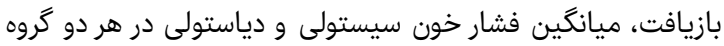

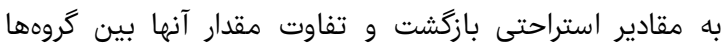

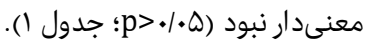

جدول ا ) مقايسه ميانكين آمارى فشار خون سيستولى و دياستولى، قبل از باز

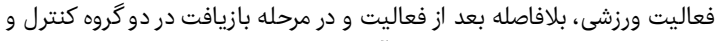

\begin{tabular}{|c|c|c|}
\hline \multicolumn{3}{|c|}{ 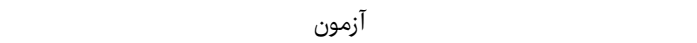 } \\
\hline كروه آزمون & كروه كنترل & متغيرها \\
\hline & & فشار خون سيستولى (ميلىمتر جيوه) \\
\hline $1 \cdot V / r r \pm \varepsilon / 1 \Lambda$ & $1 . k / k k_{ \pm 1} \cdot / k \lambda$ & ق ق قبل از فعاليت ورزشى \\
\hline$* \mid \kappa \wedge / \mu \mu \pm V / q$. & 每 & بلافاصله بعد از فعاليت \\
\hline $1 \cdot 1 / \Delta \omega \pm \Delta / \cdot \Delta$ & $1 \cdot \Delta / \mu \mu \pm V / \Delta$. & مرحله بازيافت \\
\hline & & فشار خون دياستولى (ميلىمتر جيوه) \\
\hline$V / / \& 9 \pm V / 9$. & $V Y / r Y \pm 1 \cdot / \cdot r$ & ق ق قبل از فعاليت ورزشى \\
\hline$\Lambda F / \| \pm V / F V$ & $\wedge \Lambda / q \Lambda \pm 11 / / q q$ & بلافاصله بعد از فعاليت \\
\hline$V \cdot / \mu \mu \pm V / \Delta F$ & $V Y / F F \pm q / \mu \omega$ & مرحله بازيافت \\
\hline
\end{tabular}

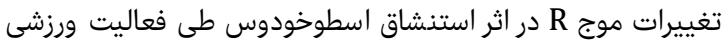

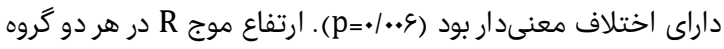

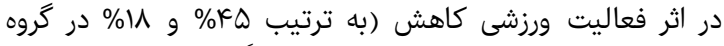

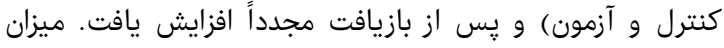

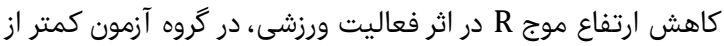

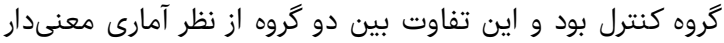

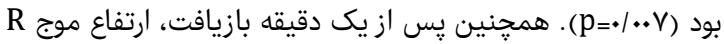

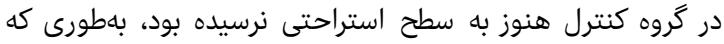

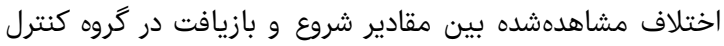

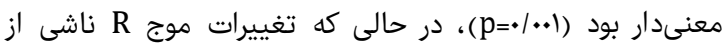

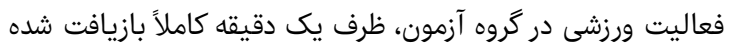

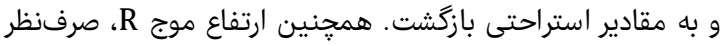

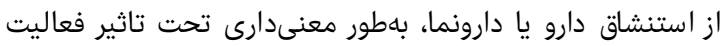

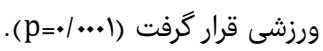

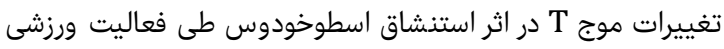

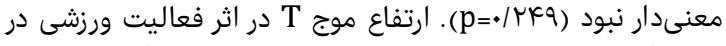

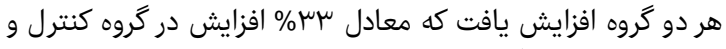

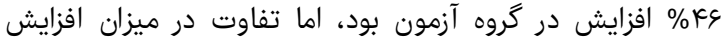

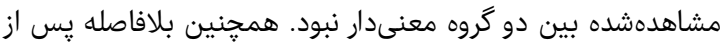

به اين ترتيب، با توجه به اثرات جسمى و روانى ذكرشده براى إن

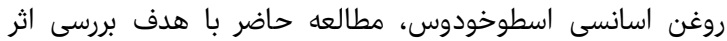

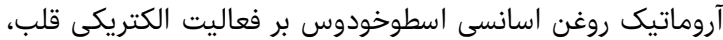
طى فعاليت ورزشى در دختران سالم انجام شد.

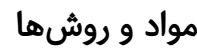

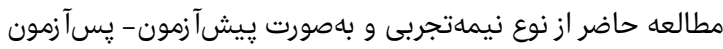

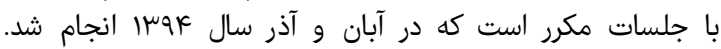

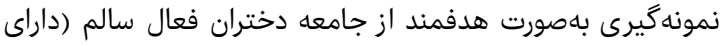

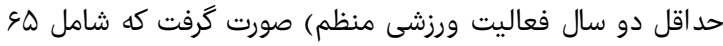

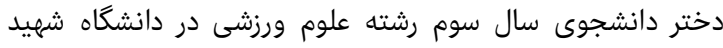

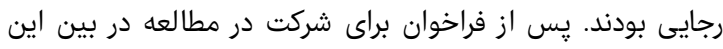

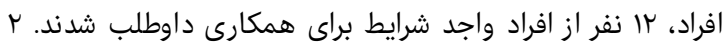

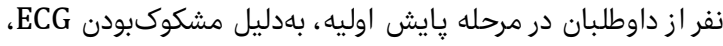

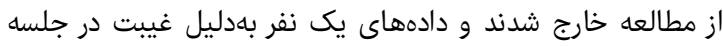

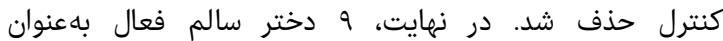

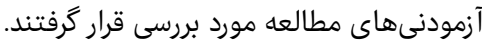

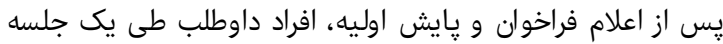

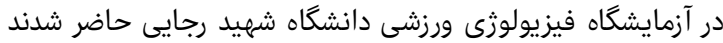

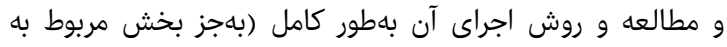

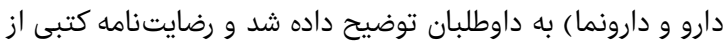

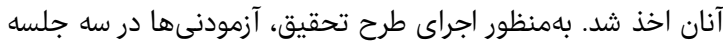

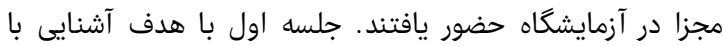

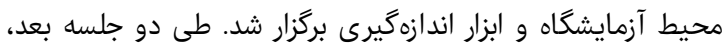

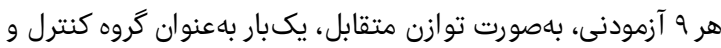

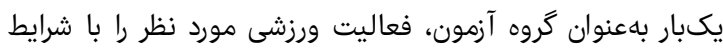

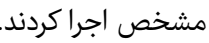

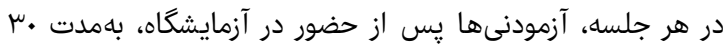

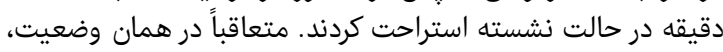

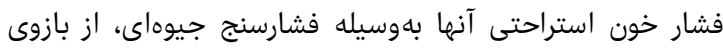

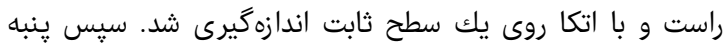

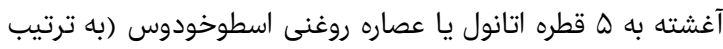

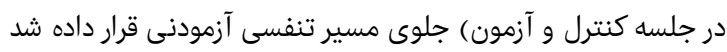

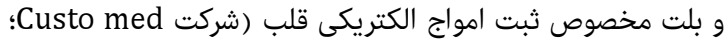

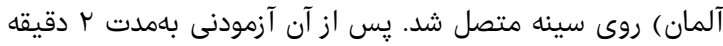

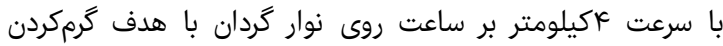

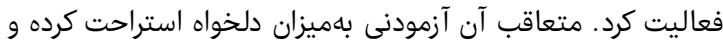

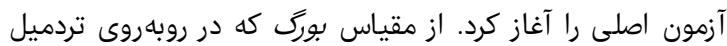

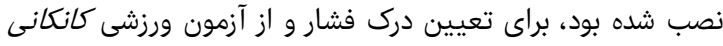

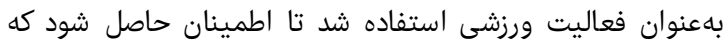

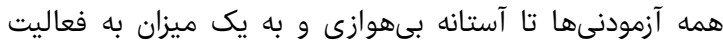

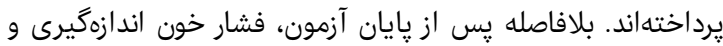

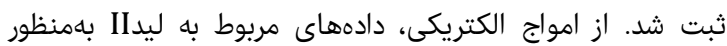

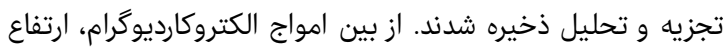

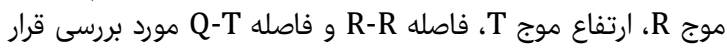

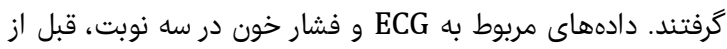

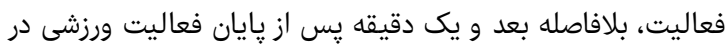
حالت نشسته (بازيافت) ثبت شد تا تغييرات آنها مورد تحليل قرار

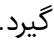

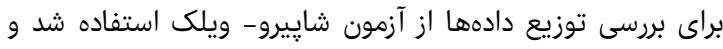

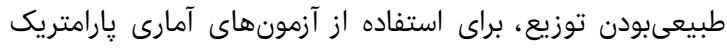

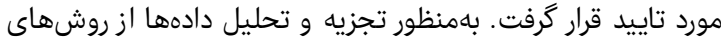


از نظر آمارى معنىدار نبود. يِيرامون تاثير استنشاق اسطوخودوس

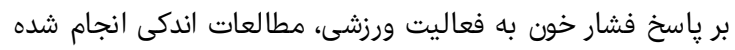

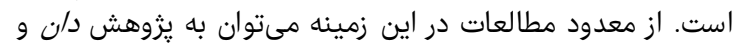

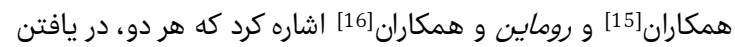

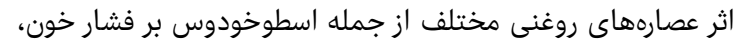

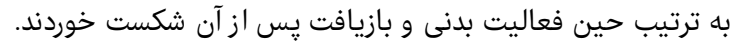

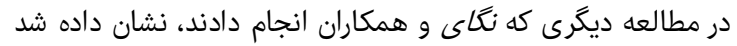

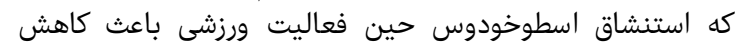

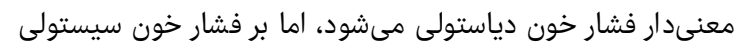

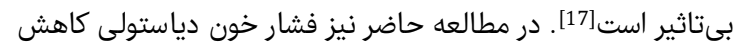

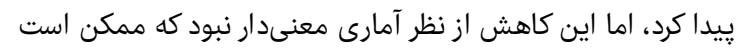

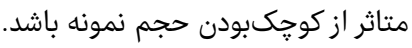

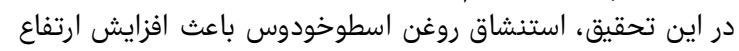

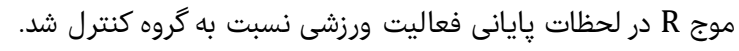

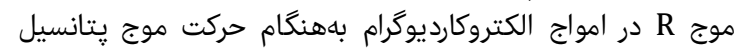

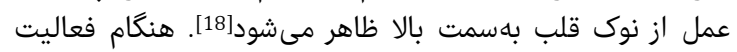

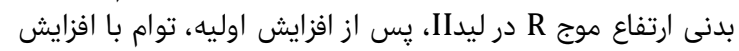

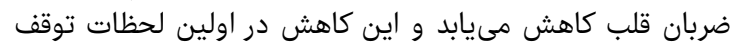

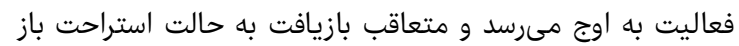

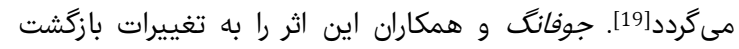

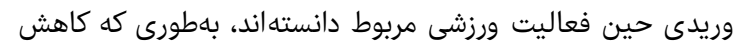

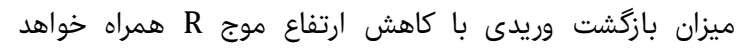

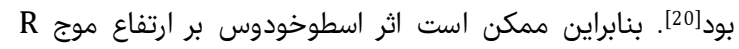

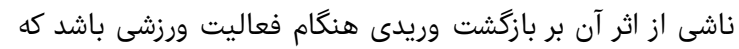

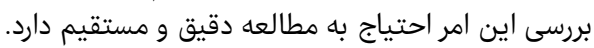

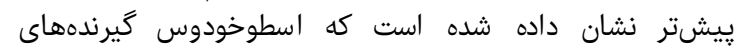

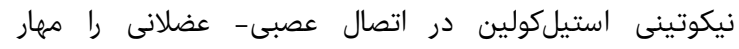

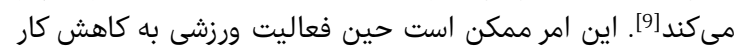

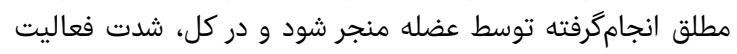

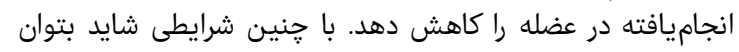

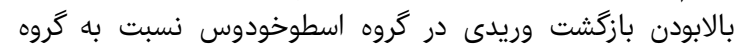

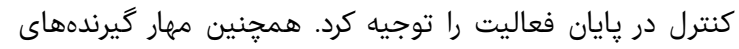

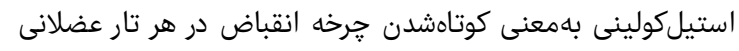

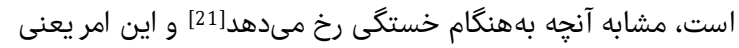

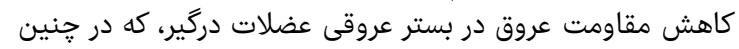

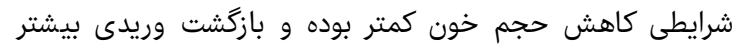

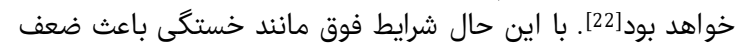

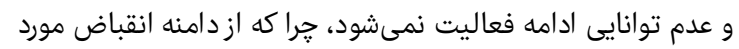

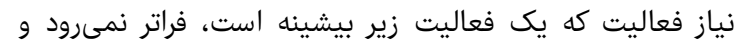

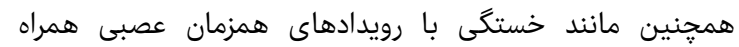

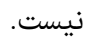

احتمال ديكر، تاثير استنشاق اسطوخودوس بر جريان خون كرونرى

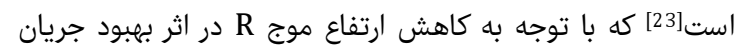

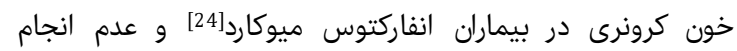

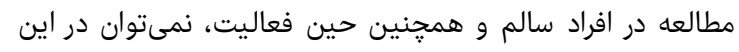

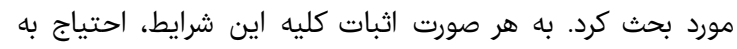

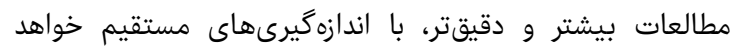

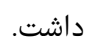

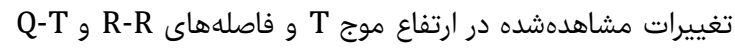

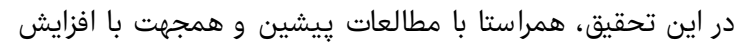

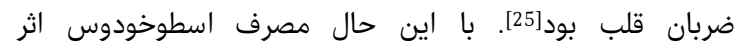
معنىدارى بر اين كاهش نداشت. نتايج بهدستآمده از مطالعه دوره سr، شماره r، بهار דوسا

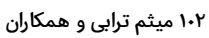

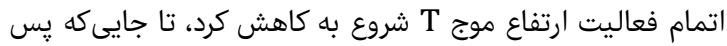

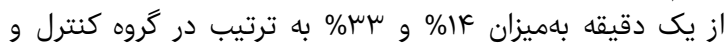

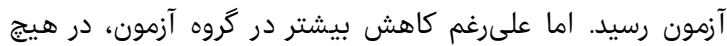

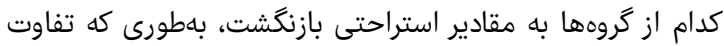

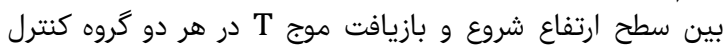

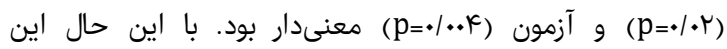

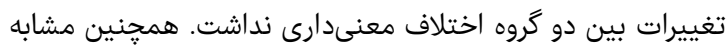

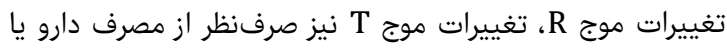

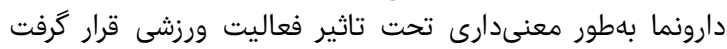

$(\mathrm{p}=\cdot / \cdots 1)$

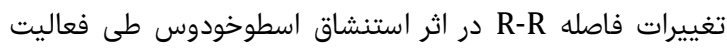

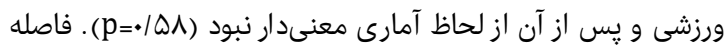

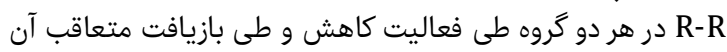

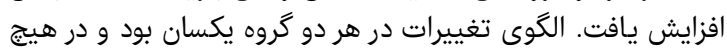

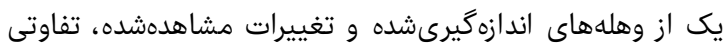

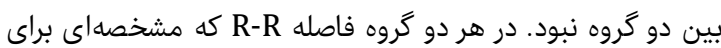

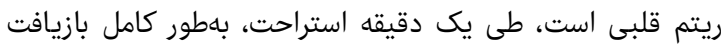

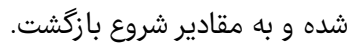
تغييرات فاصله Q-T نيز در اثر استنشاق اسطوخودوس طى فعاديت

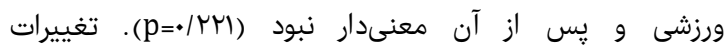

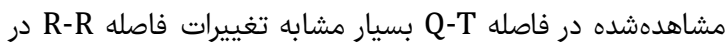

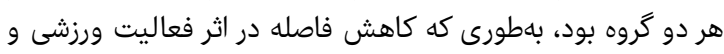

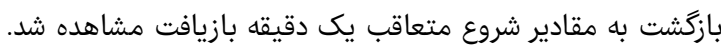

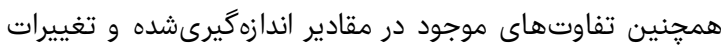

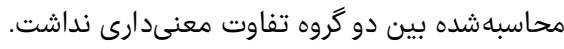

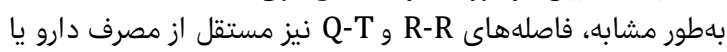

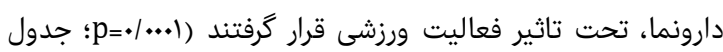

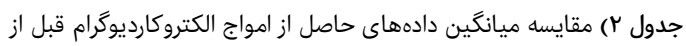

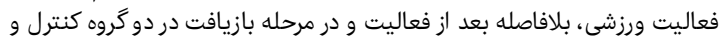

\begin{tabular}{|c|c|c|}
\hline \multicolumn{3}{|c|}{ 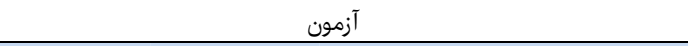 } \\
\hline گروه آزمون & تروه كنترل & متغيرها \\
\hline & & موج R (ميلىولت) \\
\hline$\cdot \mid 9 \Delta \pm \pm \cdot \cdot \cdot 1$ & $\cdot / \mathrm{V} \cdot \pm \cdot / \cdot \mathrm{r}$ & قبل از فعاليت ورزشى \\
\hline$\cdot \mid \Delta \Delta \pm \cdot / \cdot 1$ & $\cdot / 4 \wedge \pm . / \cdot 1$ & بلافاصله بعد از فعاليت \\
\hline. $\mid \varnothing 9 \pm .1 \cdot 1$ &. $\mid 9 k_{ \pm} \cdot / 1$. & مرحله بازيافت \\
\hline & & موج T (ميلىولت) \\
\hline$. / N K_{ \pm} \cdot / . . \varphi$ & $. / 1 \Delta \Delta \pm . / .19$ & قبل از فعاليت ورزشى \\
\hline$\cdot / \mu \mu \cdot \pm \cdot / \cdot 10$ & $. / r \mu r \pm \cdot \mid \cdot k q$ & بلافاصله بعد از فعاليت \\
\hline$. / Y Y Y \pm \cdot / \cdot 1$ & $. / r \cdot r \pm \cdot / \cdot r s$ & مرحله بازيافت \\
\hline & & فاصله R-R (ميلىثانيه) \\
\hline$. / 1 \& K \pm . / . K Q$ & $.|V| \pm \cdot / \cdot r r$ & قبل از فعاليت ورزشى \\
\hline$. / 1 r \cdot \pm \cdot / . .9$ & $. / 1 r Y \pm . / \cdot 11$ & بلافاصله بعد از فعاليت \\
\hline$. / 1 Q 9 \pm \cdot / \cdot \mu \mu$ & $. / 19 \Delta \pm . / . .9$ & مرحله بازيافت \\
\hline & & فاصله Q-T (ميلىثانيه) \\
\hline$\cdot / 1 \cdot k_{ \pm} \cdot 1 \cdot r r$ & $. / 11 \mathrm{~V} \pm \cdot / \cdot r s$ & قبل از فعاليت ورزشى \\
\hline 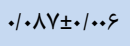 & $. / . \wedge q_{ \pm} \cdot / . r r$ & بلافاصله بعد از فعاليت \\
\hline$. / 11 r \pm \cdot / \cdot \mu$. & $. / 11 \mathrm{V \pm} \cdot / \cdot \mathrm{r \mu}$ & مرحله بازيافت \\
\hline
\end{tabular}

\section{بحث}

براساس نتايج حاصل از مطالعه حاضر، استنشاق اسطوخودوس

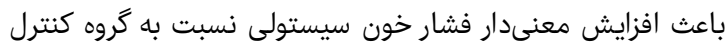

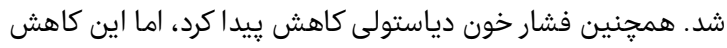
فصلنامه علمى - يُروهشى افق دانش 
تاثير روغن آروماتيك اسانسى اسطوخودوس بر فعاليت الكتريكى قلب حين فعاليت ورزشى در دختران سالم س.1

standardization and interpretation of the electrocardiogram: part I: The electrocardiogram and its technology a scientific statement from the American Heart Association Electrocardiography and Arrhythmias Committee, Council on Clinical Cardiology; the American College of Cardiology Foundation; and the Heart Rhythm Society endorsed by the International Society for Computerized Electrocardiology. J Am Coll Cardiol. 2007;49(10):1109-27.

5- Simonson E. Effect of moderate exercise on the electrocardiogram in healthy young and middle-aged men. J Appl Physiol. 1953;5(10):584-8.

6- Berka C, Johnson R, Whitmoyer M, Behneman A, Popovic D, Davis G. Biomarkers for effects of fatigue and stress on performance: EEG, P300 and heart rate variability. Proceedings of the Human Factors and Ergonomics Society Annual Meeting. 2008;52(3):192-6.

7- Estes NAM, Kloner R, Olshansky B, Virmani R. Task force 9: Drugs and performance-enhancing substances. J Am Coll Cardiol. 2005;45(8):1368-9.

8- Saki B, Paydar M, Amraei Z. The effect of garlic supplementation on aerobic performance in non-athlete men. Iran J Nutr Sci Food Technol. 2015;10(2):115-20. [Persian]

9- Cavanagh HM, Wilkinson JM. Biological activities of lavender essential oil. Phytother Res. 2002;16(4):301-8.

10- Vakilian K, Karamat A, Mousavi A, Shariati M, Ajami E, Atarha M. The effect of Lavender essence via inhalation method on labor pain. J Shahrekord Univ Med Sci. 2012;14(1):34-40. [Persian]

11- Shamsikhani S, Hekmat pu D, Sajadi Hezave M, Shamsikhani S, Khorsani S, Behzadi F. Effect of aromatherapy with lavender on quality of sleep of nursing students. Complement Med J. 2014;3(4):904-12. [Persian]

12- Khani M, Vazirian H, Jamshidi AH, Kamalipour A, Kashani L, Akhondzadeh S. Comparison of Lavandula officinalis tincture and imipramine in the treatment of mild to moderate depression: a double-blind, randomized pilot study. J Med Plants. 2002;2(2):1-8. [Persian]

13- Hajhashemi V, Ghannadi A, Sharif B. Antiinflammatory and analgesic properties of the leaf extracts and essential oil of Lavandula angustifolia Mill. J Ethnopharmacol. 2003;89(1):67-71.

14- Ziaee M, Khorrami A, Ebrahimi M, Nourafcan H, Amiraslanzadeh M, Rameshrad M, et al. Cardioprotective effects of essential oil of Lavandula angustifolia on isoproterenol-induced acute myocardial infarction in rat. Iran J Pharm Res. 2015;14(1):279-89.

15- Dunn C, Sleep J, Collett D. Sensing an improvement: an experimental study to evaluate the use of aromatherapy, massage and periods of rest in an intensive care unit. J Adv Nurs. 1995;21(1):34-40.

16- Romine IJ, Bush AM, Geist CR. Lavender aromatherapy in recovery from exercise. Percept Motor Skills. 1999;88:756-8.

17- Nagai M, Wada M, Usui N, Tanaka A, Hasebe Y. Pleasant odors attenuate the blood pressure increase during rhythmic handgrip in humans. Neurosci Lett. 2000;289(3):227-9.

18- Whyte G, Sharma S. Practical ECG for exercise science and sports medicine. Champaign, IL: Human Kinetics. 2010.

19- Mason RE, Likar I. A new system of multiple-lead exercise electrocardiography. Am Heart J. 1966;71(2):196-205.
حاضر حاكى از اثرات معنىدار اسطوخودوس بر عملكرد بطن قلب،

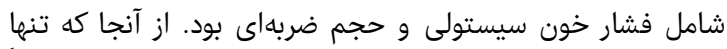

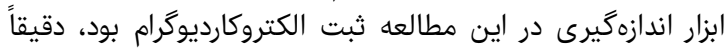

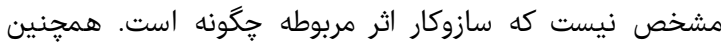

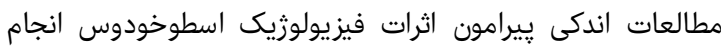

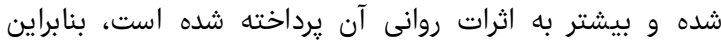

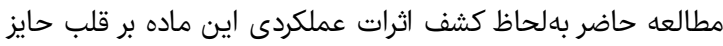

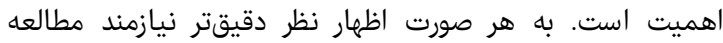

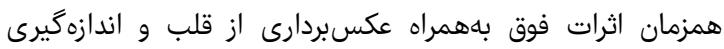
آنزيمهاى قلبى است.

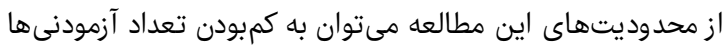

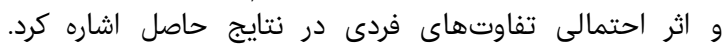

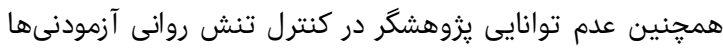

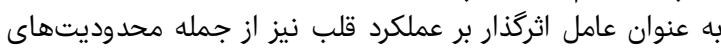

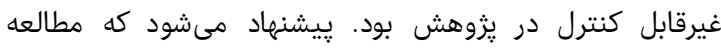

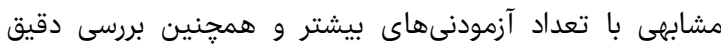

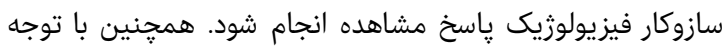

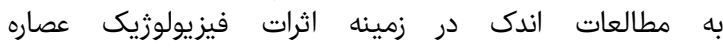

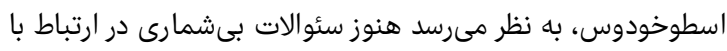

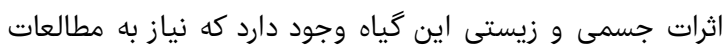
دقيق دارد.

نتيجه گيرى استنشاق اسطوخودوس بر عملكرد بطن قلب حين فعاليت ورزشى

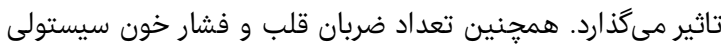
هنكام فعاليت را نيز متاثر مى سازد. تعدر.

تشكر و قدردانى: از كليه افرادى كه بهعنوان آزمودنى در اين

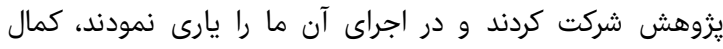

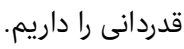
تاييديه اخلاقى: از كليه داوطلبان براى شرائ شركت در يثروهش، يِيش از

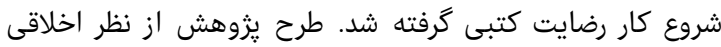

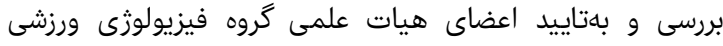

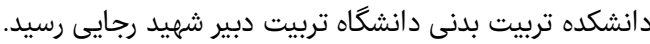

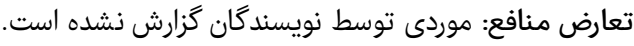

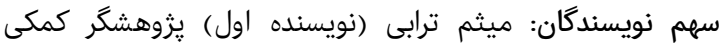

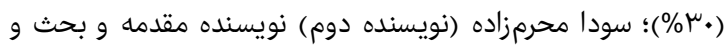

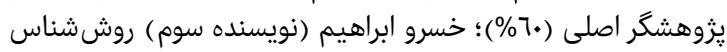

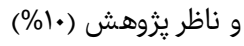
منابع مالى: اين مقاله منبع تامين مالى نداشته است.

منابع 1- Radak Z, Taylor AW, Ohno H, Goto S. Adaptation to exercise-induced oxidative stress: From muscle to brain. Exerc Immunol Rev. 2001; 7:90-107.

2- Yamamoto K, Miyachi M, Saitoh T, Yoshioka A, Onodera S. Effects of endurance training on resting and post-exercise cardiac autonomic control. Med Sci Sports Exerc. 2001;33(9):1496-502.

3- Maron BJ, Zipes DP. Introduction: eligibility recommendations for competitive athletes with cardiovascular abnormalities-general considerations. J Am Coll Cardiol. 2005;45(8):1318-20.

4- Kligfield P, Gettes LS, Bailey JJ, Childers R, Deal BJ, Hancock EW, et al. Recommendations for the 
Honjo S, et al. Relaxation effects of lavender aromatherapy improve coronary flow velocity reserve in healthy men evaluated by transthoracic Doppler echocardiography. I J Cardiol. 2008;129(2):193-7.

24- Zimetbaum PJ, Josephson ME. Use of the electrocardiogram in acute myocardial infarction. $\mathrm{N}$ Engl J Med. 2003;348(10):933-40.

25- Pourrazi H, Jafari A, Dabagh Nikoukheslat S. Cardiac biochemical, electrical and functional alterations in a group of untrained males after one bout of maximal isometric exercise. Med J Tabriz Univ Med Sci. 2010;32(1):26-32. [Persian]
20- He J, Kinouchi Y, Yamaguchi H, Miyamoto H. Exercise-induced changes in $\mathrm{R}$ wave amplitude and heart rate in normal subjects. J Electrocardiol. 1995;28(2):99106.

21- Sieck GC, Prakash YS. Fatigue at the neuromuscular junction. Branch point vs. presynaptic vs. postsynaptic mechanisms. Adv Exp Med Biol. 1995;384:83-100.

22- Hall JE. Guyton and Hall textbook of medical physiology. 13 th edition. Wynnewood, Pennsylvania: Saunders; 2015.

23- Shiina Y, Funabashi N, Lee K, Toyoda T, Sekine T, 